\title{
The use of contact lenses after refractive surgery ${ }^{\dagger}$
}

\section{R Emslie*, A Claassens* and I Walters*}

\author{
Department of Optometry, University of Johannesburg, APK, PO Box 524, Auckland Park, Johannesburg, \\ 2006 South Africa
}

<ingridwalters@webmail.co.za>

\section{Introduction}

In the last 30 years the rise of refractive surgery and the hope of permanently correcting ametropia, especially myopia, has developed and progressed greatly ${ }^{1-3}$. Like all surgical procedures, there are benefits and risks involved. In 1978, Radial Keratotomy (RK) was introduced ${ }^{1,4}$ to correct low amounts of myopia and became very popular for obvious convenience and esthetic reasons ${ }^{2}$. Radial incisions are made with a precision blade in the perilimbal cornea; the number and location of which is dependant on the amount of ametropia. These incisions alter the normal shape of the cornea causing the mid-peripheral regions of the cornea to steepen and the central optic zone area to flatten ${ }^{4,5}$. A number of studies have been done evaluating the procedure, the most commonly known one being the Prospective Evaluation of Radial Keratotomy (PERK) study ${ }^{1,6,7}$ Common visual complications arising from this incisional procedure according to the study include fluctuations in vision on a diurnal basis, glare, irregular astigmatism, epithelial defects and residual ametropia ${ }^{5,7-9}$.

The introduction of the Excimer laser led to the development of laser refractive surgery procedures such as Photorefractive keratectomy (PRK), Laser in situ keratomileusis (LASIK) and Laser epithelial keratomileusis (LASEK) ${ }^{1,10,11}$. In PRK the corneal epithelium and Bowman's layer are removed prior to the ablation of the stroma, patients have to undergo a period of recovery while the corneal epithelium regenerates $^{1,10,12,13}$. In LASIK, a microkeratome is used to create a corneal flap, which is then folded away exposing the anterior stroma. After the corneal stroma is ablated the corneal flap is replaced and left to heal ${ }^{1}$, $10,11,13$. The general recovery period for LASIK is a lot shorter than for PRK, there is also less postoperative discomfort and pain because the protective epithelial layer is intact. PRK, however, is free of the flap complications associated with LASIK and is a relatively simpler procedure ${ }^{7,10,11}$. The use of wave-front guided computerised technology ensures accurate ablation of corneal tissue and the correction of myopic, hyperopic and/or astigmatic refractive errors ${ }^{1,10,11}$. Although laser refractive procedures have fewer postsurgical complications than RK and are less common, they are not without them. Less than optimal vision can be attributed to residual ametropia (either an under or over-correction of the refractive error), regression of the ametropia, irregular astigmatism or surface irregularities, optical aberrations caused by the altered corneal shape, decreased contrast sensitivity, decentred ablation zones, flap complications and corneal haze ${ }^{3 \text {, }}$ 5, 9, 10-12, 14. Following PRK and LASIK the mid-peripheral corneal shape remains unchanged unlike RK, which is important as the visual acuity is more stable ${ }^{4,15}$. The most common complication experienced in almost all post-refractive surgery patients is dry eye. When the corneal nerves are damaged during surgery there is decreased corneal sensitivity and subsequent reduced tear production ${ }^{10,16,17}$.

Contact lens fitting in post-refractive surgery patients is challenging but often necessary to correct patients to their pre-surgical best corrected visual acuity ${ }^{3,6,10,12}$. The reduced success rate in contact lens fitting of a post-refractive surgery patient can be due to the physically altered shape of the cornea, the disrupted tear layer or psychological attributes of the patient ${ }^{1,3,6}$. Patients are often very disappointed and demotivated when having to wear contact lenses after receiving refractive surgery because often the intolerance to contact lenses or the inconvenience associated with them caused them to have the procedure in the first place $^{2}$. Patients may decide to undergo further refractive surgery in order to avoid contact lens

\footnotetext{
${ }^{\dagger}$ This paper was prepared as part of the Contact Lenses B course under the supervision of Professor WDH Gillan.

BOptom fourth - year students

Received 22 August 2007; version accepted 20 September 2007
} 
wear. The selection of contact lenses is dependent on the type of refractive procedure undergone, time after surgery, degree of corneal irregularity, mode of lens wear and best corrected spectacle acuity ${ }^{5,6}$.

\section{Therapeutic/ Bandage Contact Lenses}

Bandage contact lenses can be used to promote the healing of epithelial defects ${ }^{10,18}$ after $\operatorname{PRK}^{9,} 19$. Hydrogel lenses are very effective as bandage lenses ${ }^{19}$, although a silicone hydrogel lens is preferable with higher oxygen availability, for example Focus Night \& Day ${ }^{18}$. Bandage lenses reduce the corneal swelling after PRK. It is also used as a normal post-operative routine to encourage healing of the damaged area resulting from epithelium removal ${ }^{20}$ and to reduce pain $^{21}$. In conjunction with a bandage lens, antibiotics and steroids such as prednisolone acetate can be used to aid the healing process and thus avoid scar formation and the development of haze ${ }^{10}$. The reduction in corneal swelling confirms the function of a bandage lens as a fluid barrier. In the absence of the epithelium the fluid from the tear film can readily enter the cornea, and thus bandage contact lenses assist the endothelial pump in maintaining a normal fluid concentration ${ }^{20}$. With the occurrence of a wrinkled corneal flap after LASIK, a bandage contact lens can be used in conjunction with deionized water to eliminate the corneal wrinkles, thus eliminating the irregular astigmatism subsequently caused $^{22}$. Silicone hydrogel lenses are useful post-operatively with LASIK especially to treat recurrent corneal erosions. It is usually used for 1-3 days after surgery ${ }^{3}$. Bandage lenses are also used postLASIK to avert epithelial ingrowth and to protect the epithelium from rubbing against the eyelid ${ }^{5}$. High Dk/t bandage lenses are indicated to speed up the healing process and can be used to relieve dry eye symptoms after refractive surgery ${ }^{18}$. Complications of bandage contact lenses include microbial keratitis ${ }^{19}$, blurred vision, flap oedema, patient discomfort ${ }^{3}$, decreased tear break-up time, drying out of lenses creating discomfort ${ }^{18}$, epithelial distortion after LASIK $^{5}$ and reduced VA due to mucus accumulation beneath the lens ${ }^{21}$.

\section{Soft Contact Lenses}

Soft contact lenses are often fitted on post-refractive surgery corneas because of the relative comfort and ease of fitting associated with them ${ }^{3,6}$. In many cases of simple over or under-correction and regression of ametropia they may be adequate ${ }^{3,12}$. However, conventional soft lens designs often offer less than acceptable visual acuity and other complications. Great caution should be taken when fitting a post-RK cornea with a soft lens, often several months are needed post-surgery before the corneal shape and refraction have stabilized completely, and the incisional wounds have completely healed ${ }^{5,9,10,15}$. Even then the altered corneal shape proves extremely difficult to fit and very often vision is not stable or perfectly clear due to the flexible nature of the lens ${ }^{9,14,15}$. Loose lenses that do not compress limbal vasculature and align better with the flatter corneal contour are recommended ${ }^{6,9,15}$. Lenses of either very high water content or preferably a silicone hydrogel lens should be fitted because of the very high incidence of neovascularization development along the incision scars. Corneal oedema also occurs in post-RK patients due to endothelial cell damage and physiologically altered state of the cornea so contact lenses that maximize oxygen transmissibility should be fitted $^{5,6,9,15}$.

Soft contact lens fitting on post-PRK and LASIK eyes are far less problematic and the waiting period for fitting a soft contact lens post-surgery is shorter, usually three months are sufficient ${ }^{3,9,14,15}$. The principals of fitting are similar to those used in the fitting of a normal cornea. The lens should have a back optic zone radius 0.2 to $0.4 \mathrm{~mm}$ flatter than the flattest keratometry reading, the diameter of the lens should be 1 to 2 $\mathrm{mm}$ greater than the horizontal visible iris diameter, the lens should center well and move 1 or $2 \mathrm{~mm}$ with blinking $^{3,9}$. Care should be taken in fitting any post-refractive surgery patient with a soft lens as dry eyes, which is a common symptom, can affect the safety and comfort of lens wear ${ }^{10,14,17}$.

If a significant amount of residual astigmatism exists $(\geq 0.75 \mathrm{D})$ a toric soft lens can be fitted, however, the lenses usually do not align well and are not very stable on the surgically altered corneas. Soft lenses are also incapable of correcting for any irregular astigmatism that may be present ${ }^{3,12}$.

Patients that complain of night vision problems can be fitted with a specialized soft lens that incorporates anterior aspheric optics which will reduce some of the spherical aberrations caused by the surgery ${ }^{3,9,15}$. 


\section{Rigid Gas Permeable Lenses in post-refractive surgery fitting}

Contact lens usage after refractive surgery can provide a relatively predictable means of attaining a best corrected visual acuity and will dismiss the need for any further refractive surgery ${ }^{6}$. However, certain considerations should be kept in mind when fitting a patient who has undergone any sort of refractive surgery, this is because they experience a severe loss of corneal sensitivity and care must be taken when fitting these patients due to a lack of subjective feedback ${ }^{23}$. Rigid gas permeable (RGP) lenses are the most commonly prescribed contact lens to post-refractive surgery patients due to their high oxygen transmissibility, tear exchange properties, exceptional movement and excellent optical properties ${ }^{24}$. Their highest success rate is when fitted to corneas with a curvature no less than $38 \mathrm{D}^{25,26}$ This indicates that the normal prolate corneal shape is altered to a more oblate shape with a knee effect in the midperiphery around the surgically altered central area ${ }^{5,17,27}$. (For more information on ellipsoids and the relevant geometry thereof see Harris ${ }^{28}$.)

\section{Indications}

Indications for contact lens wear post-refractive surgery include inaccurate correction of ametropia, corneal perforation, decentration or loss of excimer flap in LASIK, irregular astigmatism and corneal ectasia $^{5}$. To fit a patient with a rigid gas permeable contact lens post-refractive surgery, it is best to wait until all corneal edema has subsided. This usually occurs after a period of 3-6 months ${ }^{6,24,29,30}$. Diurnal variations of corneal thickness can also cause increased complexity when fitting a hard contact lens. Macrae ${ }^{31}$ stated that diurnal effects are as a result of an arteriole pulse that causes size fluctuations in the eyeball, whereas Zadnik $^{9}$ believes it to be as a result of increasing edematous effects of corneal tissue throughout the day caused by hypoxia. Diurnal effects tend to subside after a period of 2-3 months ${ }^{31}$.

\section{Fitting}

To determine a midperipheral keratometric reading when calculating an initial base curve a number of methods are available. When pre-surgical keratometric readings are available a base curve made slightly flatter than these readings can be used as a starting point ${ }^{24,32,33}$. If, however, these values are unattainable, taking a midperipheral keratometric reading $3.5 \mathrm{~mm}$ above the visual axis will exhibit a fairly reliable estimate of the midperipheral radius of curvature ${ }^{24,31}$. Alternatively, the keratometric readings of the other eye may be used as a tentative starting point if it has not undergone any surgical refractive correction ${ }^{29,34}$. If central post-operative keratometric readings are taken, fit the lens approximately $2.1 \mathrm{D}$ steeper, this is another fairly reliable method of attaining an initial base curve ${ }^{21}$. Another method used is that in which the patient is told to look at four separate fixational cues placed at the outer edges of the keratometer's light source in the shape of a square. Keratometric readings are then taken whilst the patient concentrates on these fixational cues individually. A base curve is determined by taking a slightly flatter value than the mean of all four of the measured readings ${ }^{6,24,35}$. Computer generated corneal topography maps also offer information that can be used to calculate an initial base curve. McDonnel, Garbus, Caroline and Yoshinga ${ }^{36}$ stated that using information from computer generated topographical maps gives the most accurate and reliable mid peripheral and peripheral curve values. Szczokta ${ }^{37}$ recommended that an axial map representation be used instead of a tangential map; this was because she found the average plots to be more accurate thus determining a better fitting base curve. Axial maps exclude extreme curvital values and depict a more general topographical map. Topographical maps may, however, prove to be misleading when estimating and predicting the effect of the tear lens due to faults in calibration 5 .

Peripheral curves should be obtained relative to the lens position and total diameter ${ }^{9}, 38$. Due to the relatively oblate post surgical structure of the cornea a peripheral curve made $0.5-1 \mathrm{D}$ steeper than the base curve allows for better centration ${ }^{6,15,24,39}$. A well fitted peripheral curve allows adequate and desirable lens movement and tear venting. An initial trial lens with a diameter ranging from between 8-12 mm with an optic zone diameter of $2.5 \mathrm{~mm}$ less than the total diameter is commonly used ${ }^{9,40}$.

A lens power that is equal to the spherical equivalent before surgery will suffice during initial fitting ${ }^{6,9}$. Due to the central pooling between the posterior lens surface and the anterior surface of the cornea a very high plus powered tear lens is formed resulting in a very high minus powered over refraction ${ }^{5,24}$. 
Decentration occurs due to the nature of the lens to center over the steepest part of the cornea ${ }^{9,24,27}$. A high riding lens may require an increase in total diameter, an increase in base curve and an increase in lens mass by the addition of prism on the inferior lens border ${ }^{6,9,24}$. Bubbles and edematous effects are also alleviated by flattening the base curve. A low riding lens may require a lighter lens material, a decrease in total lens diameter or a decrease in optic zone diameter ${ }^{6,24}$. In post RK patients a total lens diameter change does not usually aid centration due to complications in the transitional mid-peripheral zone of the cornea. Furthermore it can be noted that fit integrity post PRK and LASIK is a lot better than that of RK due to the fact that post RK corneas have more peripheral complications ${ }^{6}$. When dealing with eccentric ablations, an 11 $\mathrm{mm}$ or greater diameter lens is used as to allow for $0.2 \mathrm{~mm}$ of the optic zone to fall outside the decentred ablation zone hence resulting in the tear lens covering the ablation ${ }^{21}$.

The ideal fit of a RGP lens post-refractive surgery is common for all types of the surgery. They all leave the central 5-6 mm of cornea considerably flatter than its periphery, which when correctly fitted with a RGP lens reveals a fluorescein pattern with moderate central pooling and an on alignment fit in the midperiphery $5,6,9,24$. This can usually be achieved by a normal spherical design, or an aspheric design for more irregular corneal astigmatism ${ }^{9,21}$. The lens should be positioned slightly superiorly and receive support from the upper lid, allowing the optic zone to center more or less over the visual $\operatorname{axis}^{5,9}$. A peripheral edge clearance of $0.12 \mathrm{~mm}$ is sufficient to allow tear exchange, eliminate peripheral adherence and reduce lid action $^{5}$.

\section{Speciality Lenses}

\section{Reverse Geometry Lenses (RGL)}

Reverse geometry lenses are fitted when the cornea is too irregular or oblate to attain a successful RGP fitting, has a low eccentricity and a non uniform optic zone. This design is usually used after refractive surgery to decrease excess central pooling, mid peripheral bearing, and to increase the stability of the lens 5 . The RGL is usually $10 \mathrm{~mm}$ in diameter with an $8 \mathrm{~mm}$ optic zone ${ }^{41}$.

The initial base curve can be determined by steepening the flattest post-operative keratometric reading by 1-1.5 D. 5, 9, 24 The base curve can also be matched to that of the RGP lens that was determined for the same post operative eye ${ }^{35}$. Axial topographical map readings are also commonly used to determine the base curve $^{5,9}$. The secondary curve or reverse curve is on average between 3-6 D steeper than the base curve as to aid in a correct fit of the oblate cornea. A peripheral curve should be chosen that produces very slight edge lift $(0.1 \mathrm{~mm})$, which when examined via the fluorescein fit pattern of the lens shows a very slight flouresence ${ }^{5,24}$. It is important to closely monitor the reverse geometry lens wearing patient's corneal physiology because with a slightly inaccurate fit adverse reactions will occur ${ }^{9}$.

\section{Hybrid Lenses}

A high-Dk hybrid lens has an inner segment that permits good vision and oxygen transmission while the skirt ensures that the lens centers well, is comfortable and is stable ${ }^{42}$. High Dk/t is very important to avoid the development of neovascularization ${ }^{3}$. For example the Softperm lens has a low Dk/t, ${ }^{3,4}$ whereas the SynergEyes lens has a Dk/t of $145 .^{43}$ The SoftPerm lens can cause corneal hypoxia which leads to corneal oedema ${ }^{4}$. SynergEyes PS has an aspheric base curve which allows for a better fit with irregular corneas ${ }^{43}$. The Total Clearance Technique ${ }^{44}$ for fitting SynergEyes PS is used to ensure apical clearance in order to avoid the formation of bubbles under the lens. When insufficient clearance occurs, the skirt curve radius can be steepened until clearance is observed. When bubbles are present with the appropriate skirt curve, the base curve should be flattened by $0.10 \mathrm{~mm}$. An over-refraction can then be performed ${ }^{44}$. Hybrid lenses can be fitted post-RK, post-LASIK and post-PRK ${ }^{9}$.

\section{Piggyback Lenses}

Piggyback lenses are hard lenses fitted on top of soft lenses. The advantage of such a lens is that the optical quality of a hard lens is combined with the comfort of a soft lens ${ }^{45}$. The hard lens can either be detachable, or a permanent fixture situated on the centre of the soft lens ${ }^{9}$. One of the problems that has arisen with the use of piggyback lenses is the reduced oxygen availability to the corneal epithelium due to 
the double lens system being formed ${ }^{9,45}$. For this reason the rigid lens should be of high gas permeability ${ }^{9}$. Piggyback lens usage proves most successful for the treatment of irregular astigmatism, corneal ectasia ${ }^{3}$, promotion of epitheliazation and alleviating RGP lens discomfort ${ }^{9}$. Focus Night $\&$ Day lenses prove to be a good choice when considering a soft base lens ${ }^{18}$, these silicone hydrogel lenses have an excellent oxygen transmissibility and are more rigid than conventional soft lenses. This makes them ideal piggyback lens material. It should be noted that these lenses tend to be less effective when fitting extremely irregular corneas $^{3}$. Piggyback lenses can be fitted to post-RK patients, this approach is aimed to better the movement and centering of the lens on the cornea ${ }^{24}$. It is undesirable to place too much negative power on the hard lens, hence if the refractive compensation requires a high negative power, it should be shared with the soft lens. However, a high plus power soft lens can be used when the upper eyelid causes unstable movement of the minus hard lens 9 .

\section{Scleral Lenses}

When faced with fitting very irregular corneas, a scleral lens is indicated ${ }^{3}$. The lens functions by vaulting the entire cornea, thus reducing the adverse effect of the corneal irregularities ${ }^{3,42}$. High Dk/t lenses are important for adequate oxygen supply ${ }^{3}$. The lens is fitted on the sclera and, when needed, toric haptic portions $^{3}$ can be used with toric sclerae. Scleral lenses are also considered for corneal ectasia. The main goal in fitting a scleral lens is corneal clearance. An overly steep fit can trap air bubbles, while a flat fit can result in discomfort. The back scleral radius must be chosen carefully to avoid excessive blanching of the conjunctiva. When PMMA lenses were solely used, fenestrations were compulsory for oxygen availability but these fenestrations increased the prevalence of bubbles. RGP scleral lenses provide more oxygen to the cornea, thus allowing sealed designs to be used with greater success. This design ensures that no bubbles get trapped under the lens. Another risk factor for bubble formation is irregular sclerae. The elimination of bubbles is a requisite for successful scleral lens fitting ${ }^{46}$.

\section{Corneal Ectasia}

Corneal ectasia is a rare post-refractive surgery complication that affects vision severely. Post surgical corneas should have a safe thickness of at least $300 \mu \mathrm{m}$, any less and corneal ectasia might develop ${ }^{10}$. It causes a progressive myopic shift that is very similar to keratoconus where the anterior cornea bulges forward $^{3}$ and the central cornea steepens and thins ${ }^{10}$. Contact lenses can be used as treatment, but if it is severe, corneal grafting is indicated ${ }^{3}$. Steele and Davidson proposed the use of a multicurve lens such as the Rose K or the Woodward design ${ }^{3}$. The Rose $\mathrm{K}$ lens is less effective where the ectasia is inferiorly displaced. Other lenses that can be used with corneal ectasia are aspheric lenses such as the Jack Allan KD, large diameter lenses such as Jack Allen S-lim ${ }^{3}$, Focus Night \& Day as a base for a piggyback lens ${ }^{18}$, the Rose K Post Graft Lens ${ }^{3}$ and the Ultravision CLPL Kerasoft ${ }^{\mathrm{TM}} 2$ which is a drape resistant soft lens ${ }^{3}$. Scleral lenses can also be fitted ${ }^{42}$. Studies done on post-PRK and LASIK corneas measuring the densities of keratocytes over five years report a steady decline in keratocyte numbers ${ }^{13}$. This may cause later development of postsurgical corneal ectasia because the keratocytes, especially those in the anterior stroma are responsible for maintaining a regular corneal structure, although there is no research supporting this theory.

\section{Conclusion}

The fitting of contact lenses post-refractive surgery requires motivated patients and an experienced practioner. The practitioner has to have an extensive knowledge of the lenses available and their desired effects. Contact lens materials and their characteristics are of great importance due to the patients' compromised corneas. Patients have to understand the limitations of contact lens wear and the benefits thereof. Patient compliance and a flexible approach will increase the chances of a desirable outcome.

\section{References}

1. Craig JP. An optometrist's personal perspective of aspects of refractive surgery. Contact Lens and Anterior Eye 2001 $2434-$ 40.

2. Gupta N, Naroo SA. Factors influencing patient choice of refractive surgery or contact lenses and choice of centre. Contact Lens and Anterior Eye 200629 17-23. 
3. Steele C, Davidson J. Contact lens fitting post-laser in situ keratomileusis (LASIK). Contact Lens and Anterior Eye 200730 84-93.

4. Schwartz CA. Speciality Contact Lenses: A Fitter's Guide. Philadelphia: WB Saunders Company, 1996.

5. Hom MM. LASIK: Clinical Co-Management. Boston: Butterworth-Heinemann, 2001.

6. Harris MG. Contact Lenses for Pre- and Post-surgery. St. Louis: Mosby, 1997.

7. Bores LD. Refractive Eye Surgery. $2^{\text {nd }}$ Ed. Blackwell Science, Inc 2001.

8. Applegate RA, Howland HC, Sharp RP, Cottingham AJ, Yee RW. Corneal aberrations and visual performance after radial keratotomy. Journal of Refractive Surgery 199814 397-407.

9. Mannis MJ, Zadnik K, Coral-Ghanem C, Kara-Jose N. Contact Lenses in Ophthalmic Practice. New York: Springer, 2004.

10. Chin JR, Chin TL. Comanagement of laser corneal refractive surgery. Contact Lens Spectrum $2006211126-31$.

11. Hanratty M. LASIK a Handbook for Optometrists Edinburgh: Butterworth-Heinemann, 2005.

12. Sonsino J. Advanced concepts in fitting contact lenses. Contact Lens Spectrum 200621 12 20-24.

13. Erie JC, Patel SV, McLaren JW, Hodge DO, Bourne WM. Corneal keratocyte deficits after photorefractive keratectomy and laser in situ keratomileusis. American Journal of Ophthalmology 2006141 800-808.

14. Yeung KK, Olson MD, Weissman BA. Complexity of contact lens fitting after refractive surgery. American Journal of Ophthalmology 2002133 607-612.

15. Efron N. Contact Lens Practice. Oxford: Butterworth-Heinemann, 2002.

16. McMonnies CW. Incomplete blinking: Exposure keratopathy, lid wiper epitheliopathy, dry eye, refractive surgery, and dry contact lenses, lid wiper epitheliopathy, dry eye, refractive surgery, and dry contact lenses. Contact Lens and Anterior Eye $20073037-51$.

17. Albietz JM, Lenton LM, McLennan SG. Dry eye after LASIK: Comparison of outcomes for Asian and Caucasian eyes. Clinical and Experimental Optometry 200588 89-95.

18. Russell GE. Bandage lenses: New opportunities in practice. Contact Lens Spectrum 2004 19 67-50.

19. Aasuri MK, Sreedar MS. Bandage contact lenses in ocular disorders. International Contact Lens Clinic 1997 $24207-211$.

20. Lattimore MR, Schallhorn SS, Lewis RB, Kaupp S. Bandage soft contact lens barrier function: a clinical research note. Contact Lens and Anterior Eye 200023 124-127.

21. Buratto L, Brint S. Custom LASIK. Thorofare: SLACK Incorporated 2003.

22. Munoz G, Alio JL, Perez-Santonja JJ, Attio WH. Successful treatment of severe wrinkled corneal flap after laser in situ keratomileusis with deionized water. American Journal of Ophthalmology 2000129 91-92.

23. Shivitz IR, Arrowsmith PN. Corneal sensitivity after radial keratotomy. Ophthalmology 1988 95 827-835.

24. Stein HA, Freeman MI, Stein RM, Maund L. Contact Lenses: fundamentals and clinical use. Thorofare: SLACK, 1997.

25. Salz JJ, Salz JM, Salz M, Jones D. Ten years' experience with a conservative approach to radial keratotomy. Refractive and Cataract Surgery 19917 12-22.

26. DePaolis MD. The role of contact lenses in the management of the radial keratotomy patient. Optometric Clinician 19944 25-34.

27. Santos VR, Waring GO, Lynn MJ, Holladay JT, Sterduto RD. Relationship between refractive error and visual acuity in the pre-operative radial keratotomy patient (PERK) study. Archive of Ophthalmology 1987 105 86-92.

28. Harris WF. Curvature of ellipsoids and other surfaces. Ophthalmic and Physiological Optics $200626497-501$.

29. Shivitz IR. Contact lenses in the treatment of patients with over-corrected radial keratetomy. Ophthalmology 198794899.

30. Shivitz IR. Fitting contact lenses after radial keratotomy. Contact Lens Forum 1988 13 38-39.

31. Macrae S, Rich L, Phillips D, Bedrossian R. Diurnal variation in vision after radial keratotomy. American Journal of Ophthalmology 1989107 262-267.

32. Ackley KD, Caroline PJ, Davis LJ. Retrospective evaluation of rigid gas permeable contact lenses on radial keratotomy patients. Optometry and Vision Science 199373 32-37.

33. Gusto J. Contact lens fitting after myopic keratomileusis. Journal of the British Contact Lens Association $19881174-75$.

34. Shivitz IA, Russel BM, Arrowsmith PN, Marks RG. Optical correction of post-operative radial keratotomy. Ophthalmology 198624 33-35.

35. Campbell MD, Caroline P. a unique technique for fitting post RK patient. Contact Lens Spectrum 199491256.

36. McDonnell PJ, Garbus JJ, Caroline P, Yoshinga PD. Computerized analysis of corneal topography as an aid in fitting contact lenses after radial keratotomy. Ophthalmology Surgeon 199223 55-59.

37. Szczokta L. Contact lenses for the irregular cornea. Contact Lens Spectrum 1998 13 6 21-27.

38. DePaolis MD, Aquavella JV, Shovlin JP. Post surgical contact lens management. In: Silbert JA ed: Anterior Segment Complications of Contact Lens Wear. New York: Churchill Livingstone, 1994.

39. Lee A, Kastl P. Rigid gas permeable contact lens fitting after radial keratotomy. Contact Lens Association of Ophthalmologists 199824 33-35.

40. Beekhuis WH, McCarey BE, van Rij G, Waring GO. Complications of hydrogel intracorneal lenses in monkeys. Archive of Ophthalmology 1989105 116-122.

41. Mathur A, Jones L, Sorbara L. Use of reverse geometry gas permeable contact lenses in the management of the post radial keratotomy patient: review and case report. International Contact Lens Clinic 199926 121-127.

42. Johar N, Watanabe R. New high-Dk hybrid lens: A successful option in fitting irregular corneas post-RK. Optometry $2007 \mathbf{7 8}$ 273.

43. Chou B. Rigid optics with soft lens comfort. Contact Lens Spectrum July 2006 48-51. 
44. DePaolis M, Beeman J, Ryan R. Fitting irregular corneas with a hybrid lens. Contact Lens Spectrum October 2006 $42-45$.

45. Weissman BA, Ye P. Calculated tear oxygen tension under contact lenses offering resistance in series: Piggyback and scleral lenses. Contact Lens and Anterior Eye 200629 231-237.

46. Pullum KW. The unique role of scleral lenses in contact lens practice. Contact Lens and Anterior Eye 199922 S26-S34. 